\title{
The anatomic basis for ultrasound in the diagnosis and treatment of styloid process-related diseases
}

\author{
Yirou Zang ${ }^{1}$, Shiyu Chen ${ }^{2}$, Guoli Zang ${ }^{2}$, Ming $\mathrm{Hu}^{2}$, Qing Xu' ${ }^{2}$ Zhubing Feng ${ }^{2}$, Ashan Pan ${ }^{3}$ \\ ${ }^{1}$ Department of Radiology, the Second Affiliated Hospital and Yuying Children's Hospital of Wenzhou Medical University, Wenzhou, China; \\ ${ }^{2}$ Department of Ultrasonography, Yueqing Affiliated Hospital of Wenzhou Medical University, Wenzhou, China; ${ }^{3}$ Department of Radiology, Yueqing \\ Affiliated Hospital of Wenzhou Medical University, Wenzhou, China \\ Contributions: (I) Conception and design: Y Zang; (II) Administrative support: G Zang; (III) Provision of study materials or patients: Y Zang, G Zang, \\ A Pan; (IV) Collection and assembly of data: M Hu, Q Xu, Z Feng; (V) Data analysis and interpretation: Y Zang, G Zang, S Chen; (VI) Manuscript \\ writing: All authors; (VII) Final approval of manuscript: All authors. \\ Correspondence to: Guoli Zang. Chief Physician, Department of Ultrasonography, Yueqing Affiliated Hospital of Wenzhou Medical University, \\ Wenzhou, China. Email: zgl6622@126.com.
}

Background: The styloid process (SP), stylohyoid ligament and lesser horn of hyoid bone together form the stylohyoid chain. Differences in the ossification degree and the connection sites of each segment of the stylohyoid chain on both sides lead to variations in the length, orientation, thickness, and straightness of SP. The incidence of elongation of the SP, known as styloid process syndrome (SPS), is around $4 \%$, with only $4 \%$ of patients showing elongation show symptoms. Computed tomography (CT) remains the firstchoice auxiliary examination for diagnosing SPS, but its performance can be affected by a variety of factors. Ultrasound can reveal the parapharyngeal space and adjacent structures, which offer high consistency with CT findings. Here, we investigated the ultrasonographic features of the SP and its adjacent structures in normal adults and assessed the clinical utility of ultrasound assessment for SP-related diseases.

Methods: With the ramus of mandible, mastoid process, SP, and salivary gland as the anatomical landmarks, ultrasonography was conducted on the parapharyngeal space in 78 healthy adults. The scans were performed along the oblique coronal section of the ramus and the cross-sectional plane between the mastoid process and ramus to visualize the SP and its adjacent structures. The SP length, the shortest distance from the SP tip to the outer edge of tonsil (SP-tonsil distance), and the distance from SP to the internal carotid artery (SP-ICA distance) were measured.

Results: SP and its adjacent structures were successfully visualized on ultrasonography in all 78 subjects. The measured SP length was $2.65 \pm 0.48 \mathrm{~cm}$. The SP-tonsil distance was $1.95 \pm 0.50 \mathrm{~cm}$. The SP-ICA distance was $0.509 \pm 0.231 \mathrm{~cm}$. The SP length and SP-tonsil distance measured by ultrasound were not significantly different from those measured by CT $(\mathrm{P}=0.071, \mathrm{P}=0.053)$. Furthermore, the SP length and SP-tonsil distance measured by ultrasound were positively correlated with CT measurements $(r=0.917, \mathrm{P}=0.071$; $\mathrm{r}=0.978, \mathrm{P}=0.053$, respectively). SP-tonsil distance was negatively correlated with SP length and SP inward deflection angle.

Conclusions: Ultrasound can accurately reveal the shape and size of SP and its adjacent structures and thus will be helpful for the diagnosis of SP-related diseases.

Keywords: Ultrasonography; applied anatomy; styloid processes (SP); related diseases

Submitted Oct 19, 2020. Accepted for publication Dec 16, 2020.

doi: 10.21037/atm-20-7781

View this article at: http://dx.doi.org/10.21037/atm-20-7781 


\section{Introduction}

The styloid process (SP) is a narrow, pointed projection that extends downwards and anteriorly from the temporal bone, with the internal carotid artery (ICA), internal jugular vein, hypoglossal nerve, and vagus nerve located medially towards it. Styloid process syndrome (SPS), also known as Eagle syndrome or elongated SP, is a condition that results in sore throat, reflex earache, head and neck pain, pharyngeal foreign body sensation, and increased salivary fluid caused by the elongated SP or the stimulation of adjacent nerve/blood vessels by abnormal location or orientation of the SP (1). The SP is difficult to observe on magnetic resonance imaging (MRI), and computed tomography (CT) remains the main diagnostic method. Unfortunately, few articles have described the application of ultrasound in SP examination, although, anatomically, ultrasound and CT images are highly consistent (2). Here, based on CT cross-sectional images, we applied ultrasound to investigate the morphology, length, and orientation of the SP and the relationships of the SP with its neighboring structures in healthy adults, with an attempt to inform the clinical diagnosis and treatment of SP-related diseases.

We present the following article in accordance with the MDAR reporting checklist (available at http://dx.doi. org/10.21037/atm-20-7781).

\section{Methods}

\section{Subjects}

A total of 78 healthy volunteers were enrolled from our center between January 2017 and December 2019. Among them there were 37 men and 41 women, aged 18-56 years (mean: $43.8 \pm 13.1$ years). The eligible subjects met the following criteria: no foreign body sensation in the pharynx; no symptoms such as tinnitus, headache, or neck pain; no palpable cord-like or bony spines in or around the tonsil fossa; and presence of a normal parapharyngeal space and nasopharynx on CT and nasal endoscopy. Exclusion criteria: history of head and neck tumors or surgical history; Mental disorders or cognitive disorders. Those who dropped out during the study. The study was approved by the Ethics Committee of Yueqing Hospital Affiliated to Wenzhou Medical University (Approval Number: YQYY201600001). All volunteers signed informed consent forms. All procedures performed in this study involving human participants were in accordance with the Declaration of Helsinki (as revised in 2013).

\section{Instrument and methods}

\section{Instruments}

The Logiq E9 (GE Healthcare) and the Q7 (Philips) were used for diagnostic ultrasonography, with the probe frequency being 1.0-5.0 and 5.0-12.0 MHz, respectively.

CT scanning was performed with a Brilliance iCT256 spiral CT machine (Philips) under the following parameters: tube voltage $120 \mathrm{kV}$, tube current $250 \mathrm{mAs}$, collimation $128 \mathrm{~mm} \times 0.625 \mathrm{~mm}$, slice thickness $0.9 \mathrm{~mm}$, reconstruction interval $0.45 \mathrm{~mm}$, rotation time $0.5 \mathrm{~s}$, and helical pitch 0.6 .

\section{Imaging examination methods Ultrasonography}

The patients were positioned in a supine position, with the head being scanned as two symmetrical hemispheres and slightly tilted backwards. The SP behind the ear and the ramus of mandible were identified on the cross-sectional sonographic images. The parotid gland on the lateral side of the parapharyngeal space and the SP, SP muscles, and internal jugular artery and vein passing through this space were identified in turn based on the gap between the mastoid process and the ramus of mandible and by using the parotid gland as the acoustic window or by placing the probe on the lower edge of the mandibular body and with the submandibular gland as the acoustic window. Based on the oblique coronal section, the overall morphology of the SP was observed, the SP length was measured, and the positional relationships between the SP and internal jugular artery and vein were observed on color Doppler flow imaging (CDFI). Subsequently, the probe was placed in the submandibular area to inspect the tonsil bed, the crosssection image showed the positional relationship between SP and tonsil, and the shortest distance between the tip or the distal part of the SP and the outer edge of the tonsil (SPtonsil distance) was measured.

\section{Ultrasound image quality evaluation}

The following image quality evaluation criteria were employed to determine the strength and weakness of the ultrasound in displaying SP and its adjacent structures: (I) complete display: the morphologies and boundaries of the structures are completely and clearly displayed; (II) partial display: about > one-half of the structures are clearly displayed; and (III) poor display: < one-half of the structures are displayed. In a double-blind set-up, two radiologists independently reviewed the sonographic images of the parapharyngeal space and its adjacent structures and completed the evaluations in accordance with the above- 

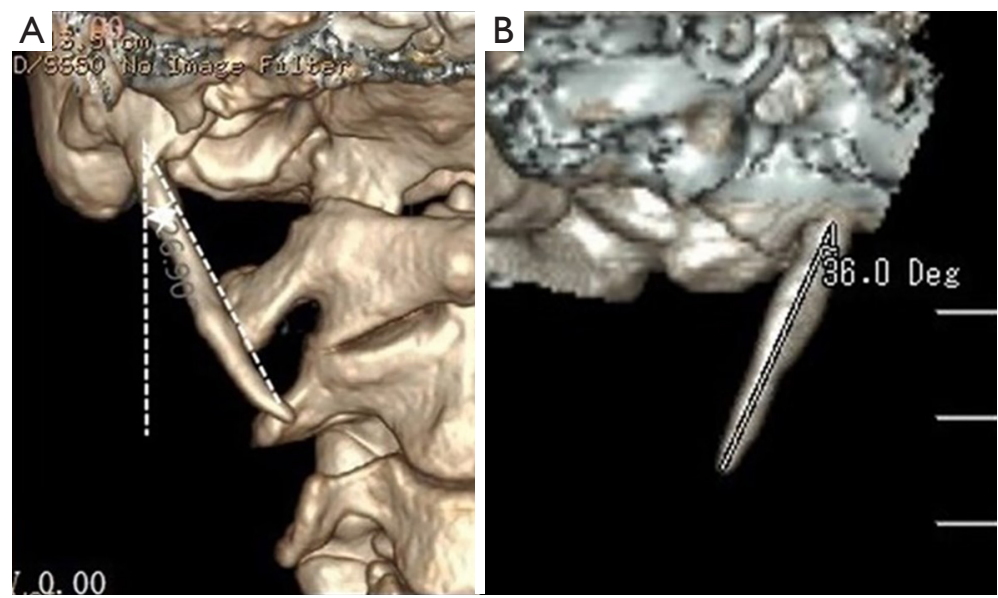

Figure 1 Volume-rendered three-dimensional reconstruction of styloid process on spiral CT. (A) Volume rendering (VR) post-processing of the CT image of the styloid process (SP) for measuring the inclination angle of the Styloid process; (B) multiplanar reconstruction (MPR) post-processing of the CT image of the SP for displaying SP length. SP, styloid process; UK, mandible; mast, mastoid process; PPS, parapharyngeal space; $\mathrm{P}$, pharyngeal cavity.

mentioned image quality evaluation criteria. If the results of the two radiologists were inconsistent, a consensus was reached after discussion.

\section{CT scans of the neck}

The subjects were asked to remove their dentures and earrings. They also were asked to be quiet and limit swallowing before the examination. The patients were asked to assume a supine position, with symmetrical body posture. The arms were hanging down, the head was in the scanning frame, and the orbitomeatal line was perpendicular to the bed. The CT scan was performed from head to toe, with the scanning range being from $20 \mathrm{~mm}$ above the frontal sinus to $20 \mathrm{~mm}$ below the front lower edge of the mandible. Techniques including volume rendering (VR) and multiplanar reconstruction (MPR) were applied. The VR and MPR images were post-processed to display the tip of the SP and measure the SP length. The post-processing of VR images revealed the course, degree of curvature, and inclination angle of the SP (Figure 1), whereas the MPR images were adjusted to the most suitable angles for measuring the SP-tonsil distance (Figure 2).

\section{Statistical analysis}

The statistical analysis was completed in SPSS 22.0 software package (IBM). The measurement data are expressed as mean \pm standard deviation. Patients were divided according to sex and the left/right sides for two independent samples $t$-test. Pearson's correlation analysis was performed on the potential correlations between SP-tonsil distance and SP length and SP inward deflection. The number of different SP morphologies was analyzed with two-sample test. A $\mathrm{P}$ value of $<0.05$ was considered to indicate a statistically significant difference.

\section{Results}

The quality data of 156 ultrasound images obtained from 78 subjects were analyzed. The SP, hyoid bone, and tonsils were completely displayed on all images (100\%), and the image quality was excellent; the parapharyngeal space was completely displayed on 151 images (96.8\%) and partially displayed on 5 images, and the image quality was good; the ICA blood flow was completely displayed on 155 images $(99.4 \%)$ and partially displayed on 1 image, and the image quality was good; and the pharyngeal wall and pharyngeal recess were completely displayed on 141 images (90.4\%) and partially displayed on 15 images, and the image quality was good. Ultrasound images with excellent and good quality could achieve accurate measurements. Unfortunately, the image quality of cranial nerves, skull base fascia, and skull base bone were poor.

Ultrasound showed that the parapharyngeal space was an inverted triangular pyramid, which could be displayed repeatedly, with a display rate of $100 \%$; its basilar part was under the petrous bone of the skull base. The deep cervical fascia at the medial boundary and the buccopharyngeal fascia, which is continued forward, had intermediate 

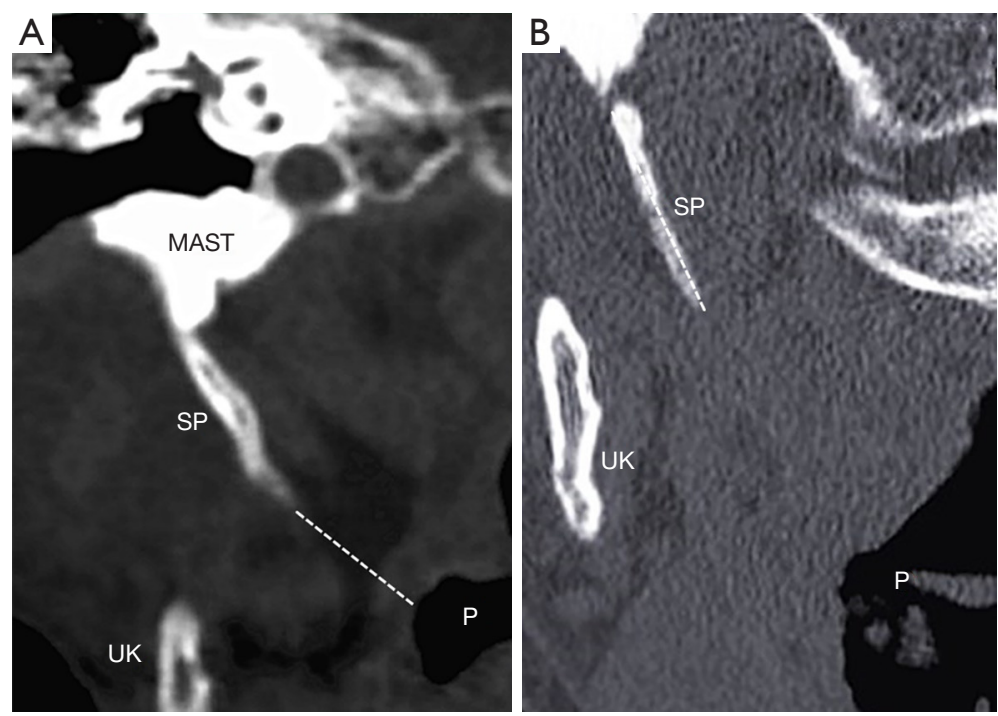

Figure 2 Multiplanar reconstruction of styloid process on spiral CT. (A) Multiplanar reconstruction (MPR) post-processing of the CT image of the SP for locating the SP tip and measuring SP-tonsil distance; (B) MPR post-processing of the CT image of the SP for measuring the SP length. SP, styloid process; UK, mandible; mast, mastoid process; P, pharyngeal cavity.
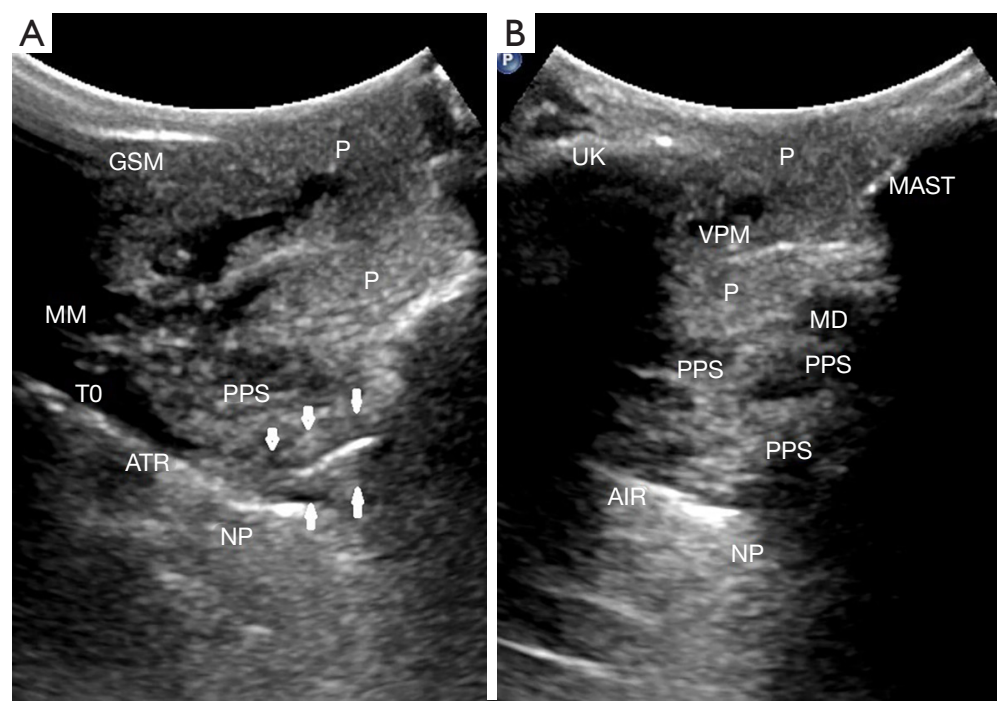

Figure 3 Sonographic evaluation of parapharyngeal space and its adjacent structures. (A) The parapharyngeal space and its adjacent structures on the oblique coronal plane of the mandibular ramus; (B) transverse section of the mandibular ramus reveals the parapharyngeal space and its adjacent structures. UK, mandible; MAST, mastoid process; P, pharyngeal cavity; GSM, submandibular gland; MD, posterior belly of the digastric muscle; RMV, retromandibular vein; PPS, parapharyngeal space; TO, tonsils; AIR, air in nasopharynx; arrows, pharyngeal recess; NP, nasopharynx.

signals, and they were tightly attached to the hypoechoic lateral walls of the nasopharynx and oropharynx, including the pharyngeal mucosa, pharyngeal recesses, and palatine tonsils (Figure 3). The anterior part of the lateral boundary was closely attached to the masseter muscle space and the submandibular gland. The posterior part of the lateral boundary included the deep lobe of the parotid gland and the posterior belly of the digastric muscle; in addition, the 

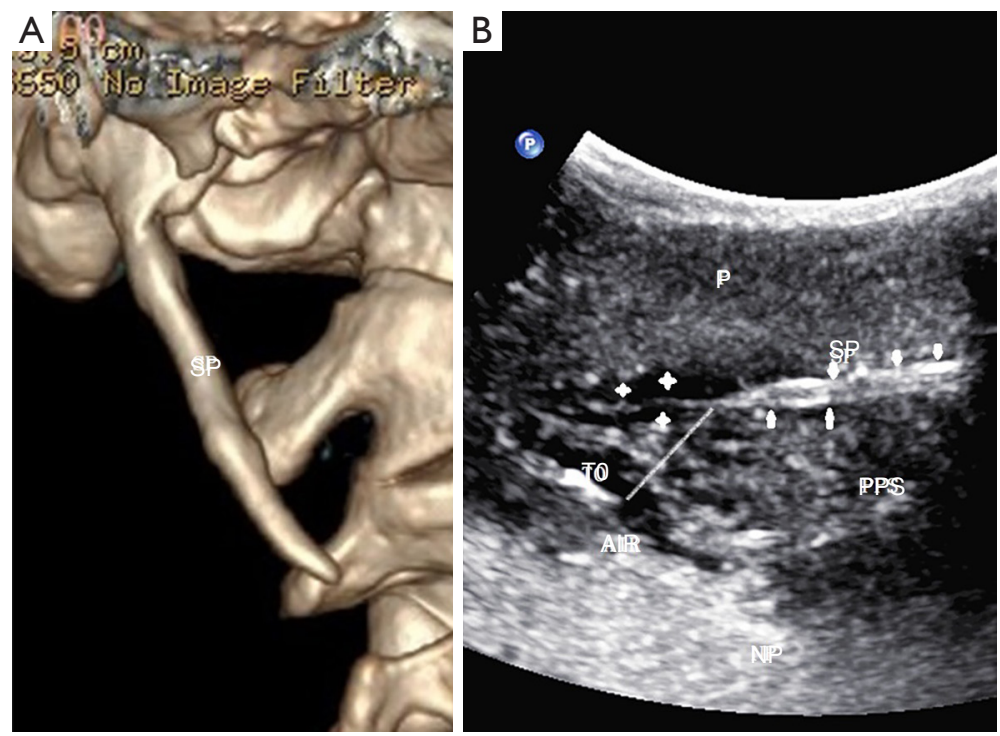

Figure 4 Spiral CT and ultrasound images of styloid process. (A) VR image of the styloid process (SP) on CT; (B) oblique coronal view of the mandibular ramus, where ultrasound reveals the long axis of the SP. P, parotid gland; SP and arrows, styloid process; asterisk, muscles attached to the SP; PPS, parapharyngeal space; TO, tonsils; line, SP-tonsil distance; AIR, air in nasopharynx; NP, nasopharynx.

Table 1 Styloid process length and distance between the styloid process and tonsil measured by ultrasound

\begin{tabular}{|c|c|c|c|c|c|c|}
\hline Group & \multicolumn{3}{|c|}{ Styloid process length $(\mathrm{cm})$} & \multicolumn{3}{|c|}{ Styloid process-tonsil distance $(\mathrm{cm})$} \\
\hline Left side & 18 & 45 & 15 & 18 & 27 & 33 \\
\hline Right side & 24 & 40 & 14 & 12 & 54 & 12 \\
\hline Total & 42 & 85 & 29 & 30 & 81 & 45 \\
\hline
\end{tabular}

SP and internal jugular artery and vein were found to be tightly attached to the lateral boundary. The three edges of the parapharyngeal space were located anteriorly, mediallyposteriorly, and laterally-posteriorly, which was consistent with the findings of anatomic dissection and CT.

On the oblique coronal plane of the mandibular branch, the SP was a hyperechoic bony structure, extending forwards and downwards from the tip of the mastoid process. With a relatively thick basilar part, the SP gradually thinned at its tip. The cross-sectional images showed a thin, round, hyperechoic structure that was adjacent to the deep parotid lobe. It could be repeatedly displayed, with a display rate of $100 \%$ (Figure 4). The SP length (the distance from the center of the SP root to the tip of the SP) as measured by ultrasound was $2.65 \pm 0.48 \mathrm{~cm}$ (range, $1.32-3.99 \mathrm{~cm}$ ), $2.68 \pm 0.42 \mathrm{~cm}$ (range, $1.38-3.99 \mathrm{~cm}$ ) for males, and $2.63 \pm 0.53 \mathrm{~cm}$ (range, $1.32-3.61 \mathrm{~cm}$ ) for females $(\mathrm{P}>0.05)$; it was $2.67 \pm 0.44 \mathrm{~cm}$ (range, $1.38-3.45 \mathrm{~cm}$ ) on the right and $2.63 \pm 0.52 \mathrm{~cm}$ (range, $1.32-3.99 \mathrm{~cm}$ ) on the left $(\mathrm{P}>0.05)$ (Tables 1,2). The SP length measured by $\mathrm{CT}$ was $2.62 \pm 0.44 \mathrm{~cm}$ (range, $1.23-3.54 \mathrm{~cm}$ ), $2.69 \pm 0.37 \mathrm{~cm}$ (range, $1.47-$ $3.54 \mathrm{~cm}$ ) for males, and $2.57 \pm 0.49 \mathrm{~cm}$ (range, $1.23-3.61 \mathrm{~cm}$ ) for females $(\mathrm{P}>0.05)$; it was $2.65 \pm 0.42 \mathrm{~cm}$ (range, $1.36-3.34 \mathrm{~cm}$ ) on the right side and $2.60 \pm 0.45 \mathrm{~cm}$ (range, $1.23-3.54 \mathrm{~cm}$ ) the left side $(\mathrm{P}>0.05)$ (Tables 3,4). The measured SP length showed no significant difference between the ultrasound group and CT group $(\mathrm{P}>0.05)$ (Table 2).

Ultrasound revealed a total of $108 \mathrm{SPs}$ with intact morphology. Among them 63 SPs (40.38\%) had linear morphology, which gradually tapered from the basilar part to the tip, forming a complete straight cone shape; 45 SPs $(28.85 \%)$ had a curved cone shape, with their morphologies also tapering from the basilar part to the tip; 43 SPs $(27.56 \%)$ were thin and long, showing segmented 
Table 2 Differences in the measurements of the styloid process length and styloid process-tonsil distance between computed tomography (CT) and ultrasound groups

\begin{tabular}{|c|c|c|c|c|c|}
\hline Parameters & $\mathrm{n}$ & Mean $(\mathrm{cm})$ & Standard deviation & $\mathrm{t}$ & $\mathrm{P}$ \\
\hline Computed tomography & 156 & 2.62 & 0.44 & & \\
\hline Ultrasound & 156 & 2.65 & 0.48 & & \\
\hline Styloid process-tonsil distance & & & & -0.798 & 0.426 \\
\hline Ultrasound & 156 & 1.95 & 0.50 & & \\
\hline
\end{tabular}

The styloid process length and styloid process-tonsil distance measured by ultrasound were not significantly different from those measured by computed tomography $(P>0.05)$.

Table 3 Differences in parameters between males and females

\begin{tabular}{|c|c|c|c|c|c|}
\hline Group & $\mathrm{n}$ & Mean & Standard deviation & $\mathrm{t}$ & $\mathrm{P}$ \\
\hline Males & 74 & 2.69 & 0.37 & & \\
\hline Females & 82 & 2.57 & 0.49 & & \\
\hline Styloid process length measured by ultrasound $(\mathrm{cm})$ & & & & 0.667 & 0.506 \\
\hline Females & 82 & 2.63 & 0.53 & & \\
\hline Styloid process-tonsil distance measured by Computed tomography $(\mathrm{cm})$ & & & & 0.992 & 0.323 \\
\hline Males & 74 & 1.98 & 0.43 & & \\
\hline Females & 82 & 1.90 & 0.55 & & \\
\hline Styloid process-internal carotid artery distance measured by ultrasound $(\mathrm{cm})$ & & & & -1.166 & 0.246 \\
\hline Males & 74 & 0.487 & 0.223 & & \\
\hline Females & 82 & 0.530 & 0.237 & & \\
\hline
\end{tabular}

No parameter showed significant difference between males and females (all $\mathrm{P}>0.05$ ).

morphologies (Figure 5); dysplasia was observed in 5 SPs (3.21\%), and no SP aplasia was detected. There was no significant difference between males and females ( $\mathrm{c} 2=0.750$, $\mathrm{P}=0.993$ ) or between the left side and right side ( $\mathrm{c} 2=0.000$, $\mathrm{P}=1.000$ ). The tips of the segmented SP swung with the rotation of head and neck, and the swing angle ranged from $5.70^{\circ}$ to $14.02^{\circ}$; dysplasia was found in two SPs, and no SP aplasia was detected.

The SP septum composed of the stylopharyngeus, styloglossus, and stylohyoid, which are attached to the
SP, was unevenly hypoechoic. SP and SP muscles divided the parapharyngeal space into two parts: the anterior parapharyngeal space (located at the anterior lateral side) and the posterior parapharyngeal space (located at the posterior medial side). The cross-sectional image passing across the mandibular branch and the mastoid showed the largest range of the posterior parapharyngeal space, which was sonographically manifested as a patchy hypoechoic area with a circular hypoechoic area. CDFI showed that these hypoechoic areas were internal and external carotid arteries. 
Table 4 Differences in parameters between the left and right-side groups of the maxillofacial region

\begin{tabular}{|c|c|c|c|c|c|}
\hline Group & $\mathrm{n}$ & Mean & Standard deviation & $\mathrm{t}$ & $\mathrm{P}$ \\
\hline Right & 78 & 2.65 & 0.42 & & \\
\hline Left & 78 & 2.60 & 0.45 & & \\
\hline Styloid process length measured by ultrasound $(\mathrm{cm})$ & & & & 0.535 & 0.593 \\
\hline Left & 78 & 2.63 & 0.52 & & \\
\hline Styloid process-tonsil distance measured by Computed tomography $(\mathrm{cm})$ & & & & -1.265 & 0.208 \\
\hline Right & 78 & 1.89 & 0.49 & & \\
\hline Left & 78 & 1.99 & 0.50 & & \\
\hline Left & 78 & 2.00 & 0.50 & & \\
\hline Styloid process-internal carotid artery distance measured by ultrasound $(\mathrm{cm})$ & & & & -0.475 & 0.635 \\
\hline Right & 78 & 0.518 & 0.225 & & \\
\hline Left & 78 & 0.501 & 0.238 & & \\
\hline
\end{tabular}

No parameter showed significant difference between left side and right side (all $\mathrm{P}>0.05$ ).
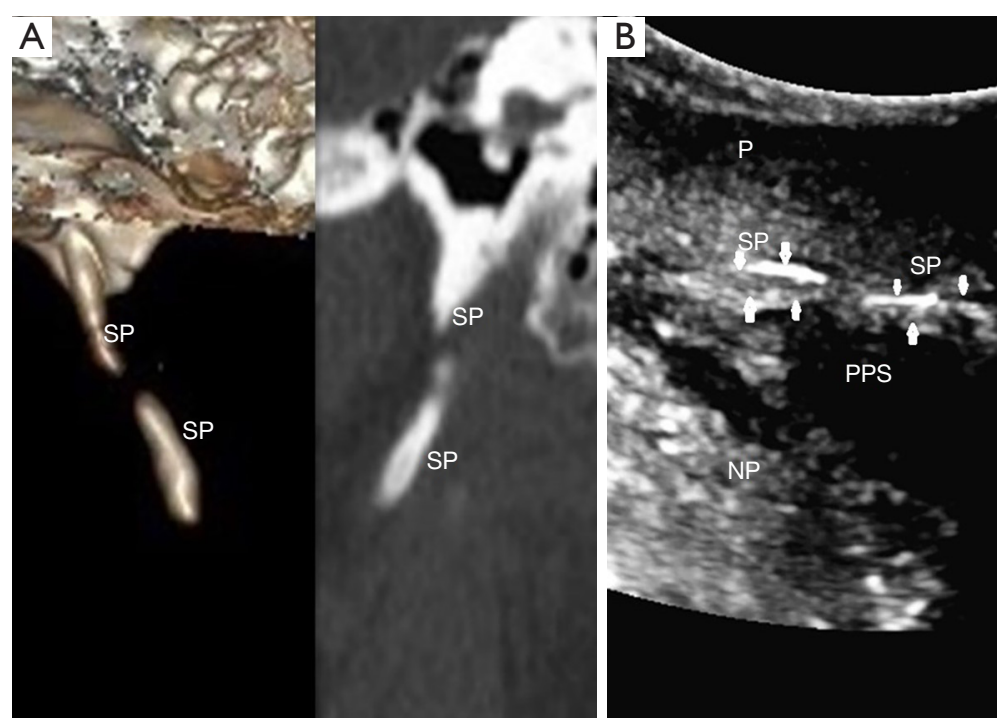

Figure 5 Spiral CT and ultrasound images of segmented styloid process. (A) CT shows a segmented styloid process (SP); (B) oblique coronal section beside the mandibular ramus shows a segmented SP. P, parotid gland; SP and arrow, styloid process; PPS, parapharyngeal space; NP, nasopharynx. 


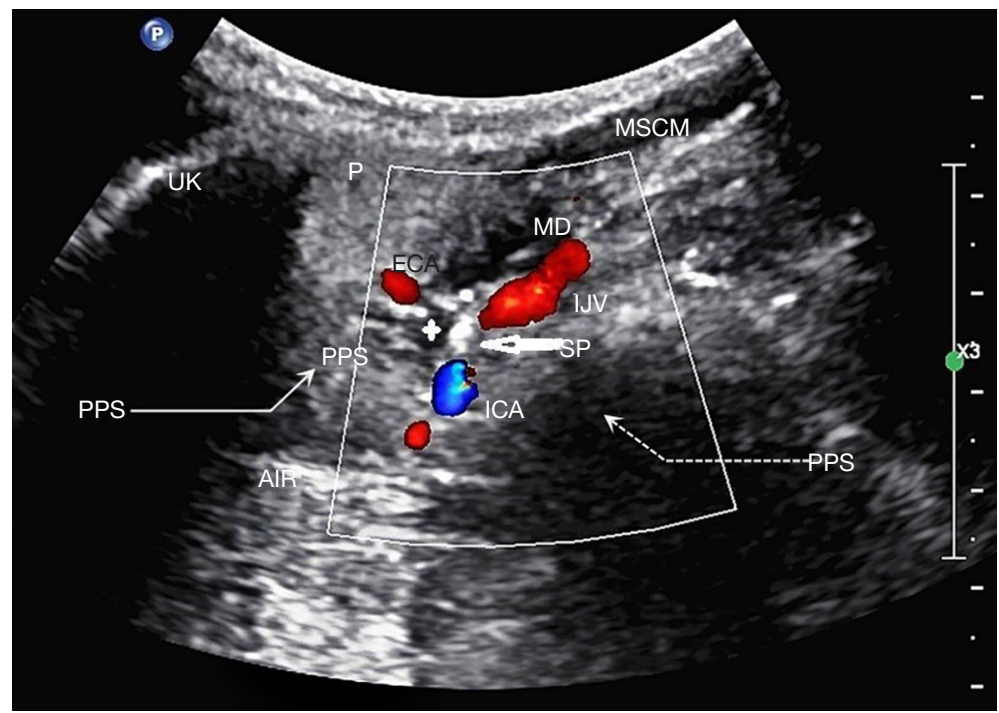

Figure 6 Through the transverse section of the mandibular ramus, CDFI on the transverse section of the parapharyngeal space shows the relationships of the styloid process (SP) with the adjacent cervical vessels. The increased inclination angle of the SP may lead to the closeness of the SP to internal jugular artery and vein. UK, mandible; MAST, mastoid process; P, parotid gland; MD, posterior belly of the digastric muscle; PPS, parapharyngeal space; solid leader line, parapharyngeal space; dotted leader line, posterior parapharyngeal space; arrow, transverse section of styloid process; asterisk, muscles attached to the SP; ICA, internal carotid artery; ECA, external carotid artery; IJV, internal jugular vein; AIR, air in nasopharynx cavity.

The tip of the SP was located between the internal and external carotid arteries. The external carotid artery crossed the SP and lay on the superficial surface, and the ICA traveled behind the SP. The SP-ICA distance measured by ultrasound was $0.509 \pm 0.231 \mathrm{~cm}$ (range, $0.136-1.445 \mathrm{~cm}$ ), $0.487 \pm 0.223 \mathrm{~cm}$ (range, $0.143 \neg-1.050 \mathrm{~cm}$ ) for males, and $0.530 \pm 0.237 \mathrm{~cm}$ (range, $0.136-3.61 \mathrm{~cm}$ ) for females $(\mathrm{P}>0.05)$; it was $0.518 \pm 0.225 \mathrm{~cm}$ (range, $0.136-1.050 \mathrm{~cm}$ ) on the right side and $0.501 \pm 0.238 \mathrm{~cm}$ (range, $0.166-1.445 \mathrm{~cm}$ ) $\mathrm{cm}$ on the left side $(\mathrm{P}>0.05)$ (Tables 3 and 4$)$. The internal jugular vein, located on the posterior lateral side of the SP, was large, oval-shaped, and hypoechoic. Ultrasound showed that the increased inward deflection of the SP could lead to the closeness of the SP to the ICA and vein (Figure 6); in addition, the decreased inward deflection of the 11 SPs resulted in the closeness or attachment of the SP to the external carotid artery (Figure 7).

The anterior parapharyngeal space was located in front of the SP and medially towards the mandibular body. The facial artery was located medially towards the anterior parapharyngeal space and ran obliquely along the lower edge of the mandibular body. The posterior belly of digastric muscle is a long bundle-shaped or elliptical, with echoes similar to those of other neck muscles. It ran through the lower part of the anterior parapharyngeal space and reached the outer side of the carotid sheath, where its superficial surface was closely attached to the deep surface of the parotid gland and the submandibular gland. The posterior belly of the digastric muscle was deeply and consistently located, forming the upper border of the triangle of the carotid artery (Figure 8).

The submandibular gland and the edge of the tongue also form a triangular area, with the palatine tonsil located in its center. The palatine tonsils were adjacent to the tongue, sonographically manifested as clear-bounded hypoechoic structures with hyperechoic gastric content. They had a striated appearance (Figure 9). The mylohyoid extended forwards and divided the submandibular gland into the body part and the uncinate process. Also, the mylohyoid and other muscles at the floor of the mouth separated the submandibular gland from the tongue. The palatine tonsils were located in anteriorly, medially, and inferiorly towards the SP (Figure 10). The SP length measured by ultrasound was $1.95 \pm 0.50 \mathrm{~cm}$ (range, $1.07-3.49 \mathrm{~cm}$ ), $1.94 \pm 0.41 \mathrm{~cm}$ (range, $1.15-2.91 \mathrm{~cm}$ ) for males, and $1.95 \pm 0.57 \mathrm{~cm}$ (range, $1.07-3.61 \mathrm{~cm})$ for females $(\mathrm{P}>0.05)$; it was $1.90 \pm 0.49 \mathrm{~cm}$ (range, $1.07-3.49 \mathrm{~cm}$ ) on the right and $2.00 \pm 0.50 \mathrm{~cm}$ (range, $1.15-3.29 \mathrm{~cm})$ on the left $(\mathrm{P}>0.05)$ (Table 1). The $\mathrm{SP}-$ 

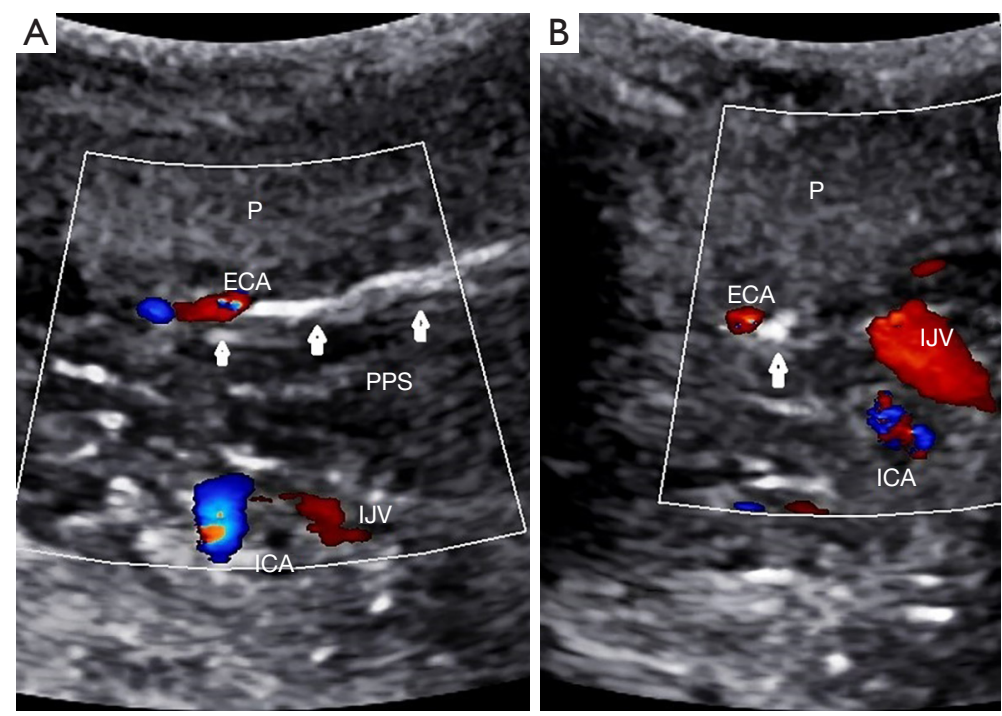

Figure 7 Ultrasound reveals the styloid process (SP) with a small inward inclination angle approaching or attaching to the external carotid artery. (A) Oblique coronal view of the mandibular ramus on ultrasound; (B) cross-sectional view of the styloid process via the transverse section of the mandibular ramus. arrows, styloid process; P, parotid gland; PPS, parapharyngeal space; ICA, internal carotid artery; ECA, external carotid artery; IJV, internal jugular vein; AIR, air in nasopharynx cavity.
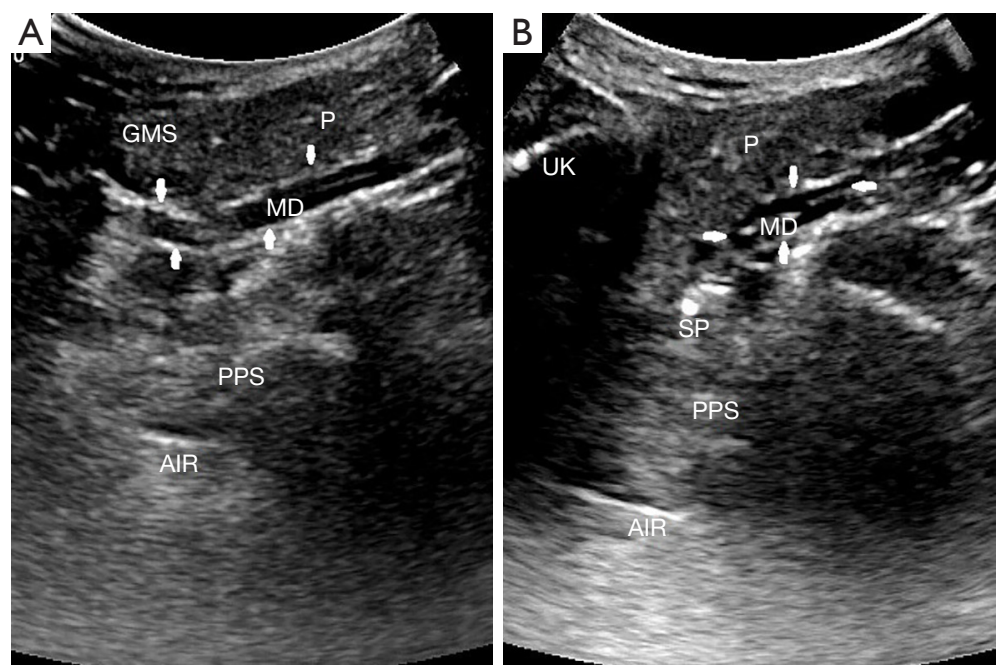

Figure 8 Sonographic evaluation of the posterior belly of the digastric muscle. (A) Oblique coronal view of the mandibular ramus shows the long axis of the posterior belly of the digastric muscle; (B) longitudinal section of the posterior belly of the digastric muscle via the transverse section of the mandibular ramus. UK, mandible; P, parotid gland; GSM, submandibular gland; MD and arrows, posterior belly of digastric muscle; PPS, parapharyngeal space; MAST, mastoid process; SP, styloid process; AIR, air in nasopharynx cavity.

tonsil distance measured by CT was $1.94 \pm 0.49 \mathrm{~cm}$ (range, $1.09-3.40 \mathrm{~cm}), 1.98 \pm 0.43 \mathrm{~cm}$ (range, $1.21-2.87 \mathrm{~cm}$ ) for males, and $1.90 \pm 0.55 \mathrm{~cm}$ (range, $1.08-3.40 \mathrm{~cm}$ ) for females $(\mathrm{P}>0.05)$; it was $1.89 \pm 0.49 \mathrm{~cm}$ (range, $1.09-3.40 \mathrm{~cm}$ ) on the right side and $1.99 \pm 0.50 \mathrm{~cm}$ (range, $1.21-3.16 \mathrm{~cm}$ ) on the left side $(\mathrm{P}>0.05)$ (Tables 3 and 4$)$. The measured SPtonsil distance showed no significant difference between the ultrasound group and CT group $(\mathrm{P}>0.053)$ (Table 2). There was a negative linear relationship between SP-tonsil distance and SP length $(\mathrm{r}=-0.693, \mathrm{P}=0.00)$, and between 

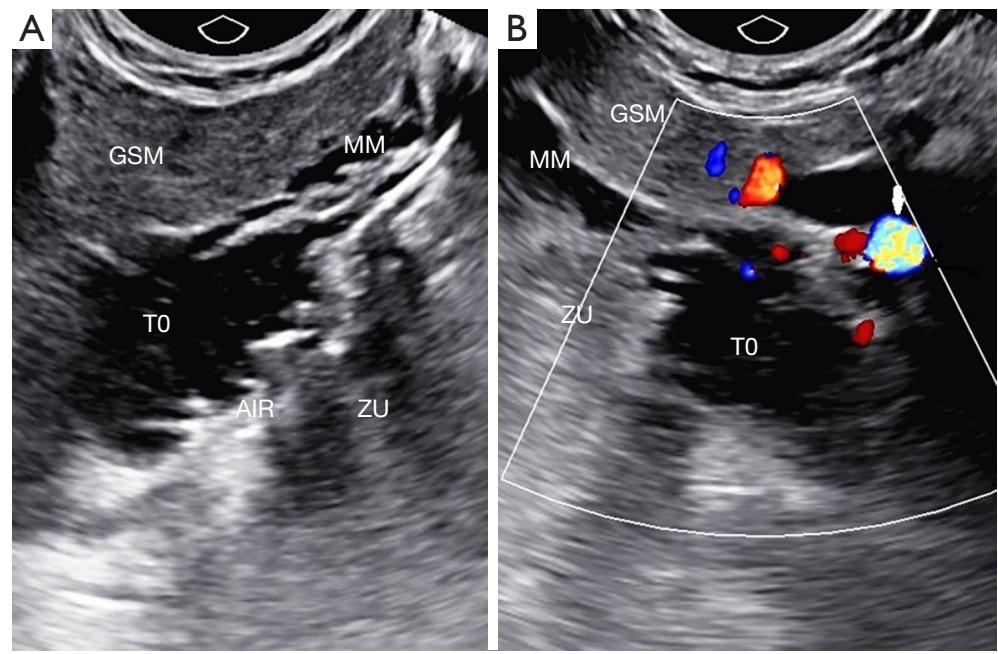

Figure 9 Sonographic evaluation of tonsils. (A) Cross-sectional view of the submandibular gland at the lower edge of the mandibular body; (B) longitudinal section of the submandibular gland at the lower edge of the mandibular body. GSM, submandibular gland; TO, tonsils; arrows, tonsillar artery; ZU, tongue; MM, mylohyoid muscle; AIR, air between tonsil and tongue.

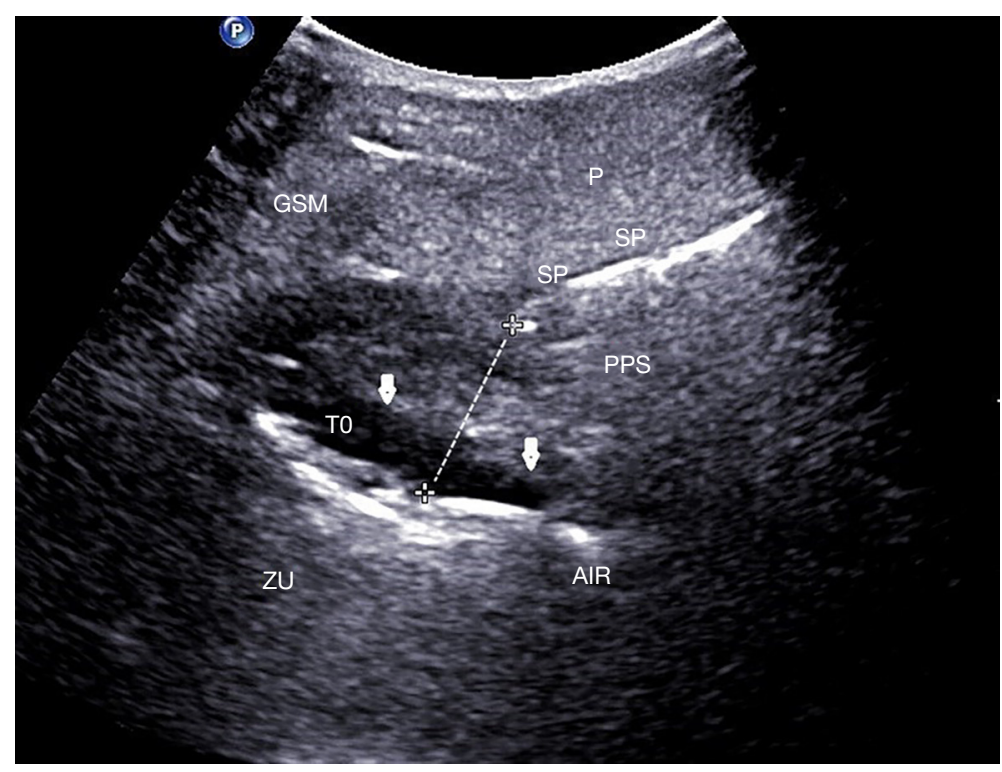

Figure 10 Measurement of the styloid process-tonsil distance on the oblique coronal plane of the mandibular ramus. cursor and dotted line, styloid process-tonsil distance; SP, styloid process; TO and arrows, tonsils; P, parotid gland; GSM, submandibular gland; PPS, parapharyngeal space; ZU, tongue; air, air in nasopharynx cavity.

SP-tonsil distance and the inward deflection angle of the SP (measured by CT) $(r=-0.341, \mathrm{P}=0.00)$.

\section{Discussion}

Currently the diagnosis of SPS is mainly based on the palpation of the SP in the tonsil fossa, the triggered pain, and the abnormal length and position angles of the SP on imaging (3). However, the development of the SP varies among individuals, and there is no consensus on the length and angles of the SP; as a result, no widely recognized criteria for the imaging diagnosis of SPS exists. 
Anatomically, SP is slender and cylindrical. It starts from below the tympanic part of the temporal bone and above the medial and upper side of the mastoid tip, extending forwards and downwards. Its root is surrounded by the SP sheath, and the stylopharyngeus is attached to the medial and posterior sides of the root, with the tonsils located anteriorly, medially, and inferiorly towards the SP. The stylohyoid is attached to the posterior and lateral sides of the middle of SP, and the stylopharyngeus is attached to the anterior side. The SP, stylohyoid ligament, lesser cornu of hyoid bone, and body of hyoid bone together form the stylohyoid chain, with the SP tip facing the tonsillar fossa. There are two special types of abnormal SP morphologies: ossification of the stylohyoid ligament and SP bone nonunion. During the development of SP, its root and body fuse into a complete process, and its tip is gradually ossified and elongated along the stylohyoid ligament. Depending on the ossification process, the SP has differences in length, thickness, straightness, and inclination. If the ossification during the fusion of the body and root of SP is incomplete, a fibrous connection will form between them, which is known as segmented SP. In individuals with segmented SP, the SP tip swings with the rotation of the head and neck and may compress the ICA and stimulate the nerves at certain positions, leading to the occurrence of symptoms (4). In a small sample of our current study, we found that, in individuals with segmented SP, the SP tip swung towards the lateral wall of the pharynx when the head and neck rotated from the neutral position, and the maximum swing angle was $14.2^{\circ}$. The SP tip may be gradually ossified and elongated along the stylohyoid ligament, which may lead to stylohyoid ligament ossification once a long SP is completely ossified. If the stylohyoid chain is ossified or if the SP is too long, resulting in increased inward deflection, the SP-tonsil distance will be shortened, and a bony protrusion can be palpated around the tonsil fossa. While the complete-type SPS has been well reported, few articles have described the segmented and dysplastic types.

Research on the morphology of SP is of great significance for the diagnosis and treatment of SPS. The $\mathrm{SP}$ is an important bony landmark of the parapharyngeal space. There are external carotid artery, internal jugular vein, and facial nerves in the superficial surface of the SP. The SP itself has the function of marking and protecting the large blood vessels and nerves in its deep surface. Thus, it is the "golden key" to identify nerves and blood vessels on CT and MRI images (5). Ultrasound can perfectly display the entire parapharyngeal space, which has the shape of an inverted triangular pyramid. Also, it can clearly show the full picture of the SP and its spatial relationships with adjacent structures, and measure the SP length. It has been reported that the length of the normal SP ranges between 2.5 and $3.0 \mathrm{~cm} \mathrm{(6),} \mathrm{and} \mathrm{most} \mathrm{investigators} \mathrm{believe} \mathrm{that} \mathrm{a}$ diagnosis of SPS can be made if the SP length exceeds $3.5 \mathrm{~cm}$. Maher et al. reported an SPS patient treated with ultrasound-guided peristyloid steroid injection, in whom ultrasound dynamically revealed that SP was located about $2.0 \mathrm{~cm}$ below the skin, appeared as a long thin hyperechoic structure, accompanied by an acoustic shadow; color Doppler displayed that the internal jugular vein and internal carotid artery were slightly deeper than SP (7). $\mathrm{Li}$ et al. reported that, among the imaging techniques for SPS, CT was the standard method and MRI could be used for locating adjacent vessels; in contrast, ultrasound could display the elongated styloid process and diagnose SPS, reduce radiation dose, and largely avoid the use of costintensive diagnostic techniques (8). As ultrasound can reveal bone, soft tissue, and peripheral blood vessels in a real-time manner, Liu et al. believed ultrasound-guided glossopharyngeal nerve block via SP was a safe, radiationfree, repeatable, convenient, and effective treatment as it allowed the direct observation of the diffusion of drugs and enabled the operator to effectively avoid important structures (e.g., blood vessels) and thus reduced the occurrence of complications (9).

In our current study, the ultrasound-measured SP length was $2.65 \pm 0.48 \mathrm{~cm}$ (range, $1.32-3.99 \mathrm{~cm}$ ), while the CTmeasured SP length was $2.62 \pm 0.44 \mathrm{~cm}$ (range, $1.23-3.54 \mathrm{~cm}$ ). More specifically, the ultrasound-measured SP length was $2.68 \pm 0.42 \mathrm{~cm}$ (range, $1.38-3.99 \mathrm{~cm}$ ) in males and $2.63 \pm 0.53 \mathrm{~cm}$ (range, $1.32-3.61 \mathrm{~cm}$ ) in females, and was $2.67 \pm 0.44 \mathrm{~cm}$ (range, $1.38-3.45 \mathrm{~cm}$ ) on the right side and $2.63 \pm 0.52 \mathrm{~cm}$ (range, $1.32-3.99 \mathrm{~cm}$ ) on the left side. In contrast, the CT-measured SP length was $2.69 \pm 0.37 \mathrm{~cm}$ (range, $1.47-3.54 \mathrm{~cm}$ ) in males and $2.57 \pm 0.49 \mathrm{~cm}$ (range, $1.23-3.31 \mathrm{~cm}$ ) in females, and was $2.65 \pm 0.42 \mathrm{~cm}$ (range, $1.36-3.34 \mathrm{~cm}$ ) on the right side and $2.60 \pm 0.45 \mathrm{~cm}$ (range, $1.23-3.54 \mathrm{~cm}$ ) on the left side. These measurements were consistent with those reported in the literature, there was a close correlation between ultrasound and CT in determining the styloid process length. It was found that all the length of the normal SP measured by ultrasound was longer than those measured by CT, which might be explained by the low resolution of X-ray in displaying cartilage in enlarged SP. Research on the morphology of SP is of great significance for the diagnosis and treatment of 
SPS. But The accuracy of Ultrasound Measurement of SP is related to the experience of Sonographer.

Located between the ICA and the external carotid artery, the distal end of the SP extends inwards, forwards, and downwards $(10,11)$. As shown in our current study, ultrasound could clearly reveal the positional relationship between the SP and blood vessels in the neck. The tip of the SP was located between the internal and external carotid arteries. The external carotid artery crossed the SP and lay on the superficial surface, and the ICA traveled behind the SP. Ultrasound showed that the increased inward deflection of the SP could lead to the closeness of the SP-ICA and vein. Renard et al. (12) evaluated the distance between the SP and the ICA and concluded that the shortened distance between the SP or hyoid bone and ICA might be an important cause of ICA dissection. The vascular type of SPS is also associated with the hemodynamic changes following the compression of adjacent blood vessels by SP. In our current study, the SP-ICA distance measured by ultrasound was $0.44 \pm 0.25 \mathrm{~cm}$. Ultrasound revealed that the inward deflection of the SP was small in some cases, and the SP was obviously close to the external carotid artery; within a certain length, the SP could compress the external carotid artery and its branches, affecting blood circulation and causing pain at the inferior orbital area, temporal area, ears, and occipital areas. The pain can radiate upwards from the mandibular angle to the head, neck, or face. Sometimes the patients may experience neurasthenia symptoms such as tinnitus, salivation, and insomnia.

The palatine tonsils are located anteriorly, medially, and inferiorly towards the SP and in the tonsillar fossa between the palatoglossal arch and palatopharyngeal arch. The oblique section of the submandibular gland is the best way for obtaining an optimal sonographic image of tonsils. Sonographically, normal tonsils have an oval shape and are evenly hypoechoic; they are the largest in shape during childhood and will shrink with age, which manifests as smaller size, increased calcification, and less blood flow. A cross-sectional ultrasound scan through the submandibular gland can clearly display the dynamic relationship between the SP tip and the tonsils, and the SP-tonsil distance can be used in the diagnosis of SPS. It was reported that the SPtonsil distance was significantly shorter in SPS patients (12.7 $\mathrm{mm})$ than in asymptomatic controls $(24.8 \mathrm{~mm})(\mathrm{P}<0.0005)$ and in patients with glossopharyngeal neuralgia $(21.4 \mathrm{~mm})$ $(\mathrm{P}=0.027)$ (13). In our current study, the SP-tonsil distance measured by ultrasound was $1.95 \pm 0.50 \mathrm{~cm}$ (range, $1.07-3.49$ $\mathrm{cm}$ ), while that measured by CT was $1.94 \pm 0.49 \mathrm{~cm}$ (range,
$1.09-3.40 \mathrm{~cm}$ ). There was a positive linear correlation between these two measurements. In addition, we found that SP-tonsil distance was negatively correlated with the SP length and the inward deflection angle of the SP. As the SP became longer and the inward deflection angle increased, the SP-tonsil distance was shortened.

CT and MRI are sometimes less helpful in determining the origin of a lesion near the interface between the parotid gland and parapharyngeal space, especially when the lesion is deep into parotid a gland that has protruded into the parapharyngeal space anteriorly towards the SP. Traditionally, the signs of SP displacement and cervical sheath vessel displacement may solve some of the above problems. However, the SP is difficult to observe on MRI; in addition, the lesion often compresses and displaces the cervical blood vessels, making it difficult to identify the cervical sheath vessel displacement. As positioning errors lead to deviations in recognition, other reliable landmarks are urgently required. Since the SP and the posterior belly of the digastric muscle are located at the interface between the parotid gland and the parapharyngeal space, ultrasound can reliably reveal the SP and digastric muscle; therefore, these two structures can be used as a landmark for identifying the gap where deep cervical lesions originate (14). The position of ultrasonic anatomy of the parapharyngeal space is more important and it is worth to be used widely in clinical practice.

SPS is a disease caused by multiple factors. On the basis of digital pharyngeal examination, CT is the preferred auxiliary examination for diagnosing SPS. Ultrasound can dynamically display the anatomical structures of parapharyngeal space, SP, tonsils, posterior belly of the digastric muscle, and internal jugular arteries and veins in real-time. It can simply and clearly reveal the overall morphology of the SP and can visualize the tendons and ligaments attached to the SP along with the relationships of the SP with its surrounding structures. This technique of ultrasound is easy to master. Therefore, ultrasound can be used as an important supplement in the diagnosis of SPS. Meanwhile, ultrasound-guided nerve block via the styloid process is a safe, radiation-free, repeaBcl-2, convenient, and effective treatment. It can provide a treatment option for patients with SPS.

\section{Acknowledgments}

Funding: Supported by Wenzhou Municipal Science and Technology Bureau Program (Y20180752). 


\section{Footnote}

Reporting Checklist: The authors have completed the MDAR reporting checklist. Available at http://dx.doi.org/10.21037/ atm-20-7781

Data Sharing Statement: Available at http://dx.doi. org/10.21037/atm-20-7781

Conflicts of Interest: All authors have completed the ICMJE uniform disclosure form (available at http://dx.doi. org/10.21037/atm-20-7781). The authors have no conflicts of interest to declare.

Ethical Statement: The authors are accountable for all aspects of the work in ensuring that questions related to the accuracy or integrity of any part of the work are appropriately investigated and resolved. The study was approved by the Ethics Committee of Yueqing Hospital Affiliated to Wenzhou Medical University (Approval Number: YQYY201600001). All volunteers signed informed consent forms. All procedures performed in this study involving human participants were in accordance with the Declaration of Helsinki (as revised in 2013).

Open Access Statement: This is an Open Access article distributed in accordance with the Creative Commons Attribution-NonCommercial-NoDerivs 4.0 International License (CC BY-NC-ND 4.0), which permits the noncommercial replication and distribution of the article with the strict proviso that no changes or edits are made and the original work is properly cited (including links to both the formal publication through the relevant DOI and the license). See: https://creativecommons.org/licenses/by-nc-nd/4.0/.

\section{References}

1. Cullu N, Deveer M, Sahan M, et al. Radiological evaluation of the styloid process length in the normal population. Folia Morphol (Warsz) 2013;72:318-21.

2. Singh M, Chin KJ, Chan VW, et al. Use of sonography for airway assessment: an observational study. J Ultrasound

Cite this article as: Zang $\mathrm{Y}$, Chen S, Zang G, Hu M, $\mathrm{Xu} \mathrm{Q}$, Feng Z, Pan A. The anatomic basis for ultrasound in the diagnosis and treatment of styloid process-related diseases. Ann Transl Med 2020;8(24):1666. doi: 10.21037/atm-20-7781
Med 2010;29:79-85.

3. Muñoz-Leija MA, Ordóñez Rivas FO, Barrera-Flores FJ, et al. A proposed extension to the elongated styloid process definition: A morphological study with high-resolution tomography computer. Morphologie 2020;104:117-124.

4. Westbrook AM, Kabbaz VJ, Showalter CR. Eagle's syndrome, elongated styloid process and new evidence for pre-manipulative precautions for potential cervical arterial dysfunction. Musculoskelet Sci Pract 2020;50:102219.

5. Badhey A, Jategaonkar A, Anglin Kovacs AJ, et al. Eagle syndrome: A comprehensive review. Clin Neurol Neurosurg 2017;159:34-38.

6. Eagle WW. Elongated styloid process; symptoms and treatment. AMA Arch Otolaryngol 1958;67:172-6.

7. Maher T, Shankar H. Ultrasound-Guided Peristyloid Steroid Injection for Eagle Syndrome. Pain Pract 2017;17:554-7.

8. Li Z, Hua Y, Yang J, Li J. Ultrasound Evaluation of Transient Ischemic Attack Caused by Styloid Process Elongation: A Case Report. Front Neurol 2019;10:26.

9. Liu Q, Zhong Q, Tang G, He G. Ultrasound-guided glossopharyngeal nerve block via the styloid process for glossopharyngeal neuralgia: a retrospective study. J Pain Res 2019;12:2503-10.

10. Eraslan C, Ozer MA, Govsa F, et al. Relationship of stylohyoid chain and cervical internal carotid artery detected by 3D angiography. Surg Radiol Anat 2017;39:897-904.

11. Amorim JM, Pereira D, Rodrigues MG, et al. Anatomical characteristics of the styloid process in internal carotid artery dissection: Case-control study. Int J Stroke 2018;13:400-5.

12. Renard D, Azakri S, Arquizan C, et al. Styloid and hyoid bone proximity is a risk factor for cervical carotid artery dissection. Stroke 2013;44:2475-9.

13. Pokharel M, Karki S, Shrestha I, et al. Clinicoradiologic evaluation of Eagle's syndrome and its management. Kathmandu Univ Med J (KUMJ) 2013;11:305-9.

14. Kim SD, Loukas $M$. Anatomy and variations of digastric muscle. Anat Cell Biol 2019;52:1-11.

(English Language Editor: J. Gray) 